\title{
La canine.
}

\section{Première partie : \\ Définition et évolution durant l'Ère secondaire}

\section{RÉSUMÉ}

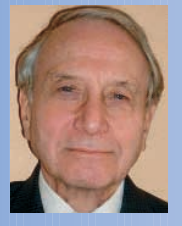

Jean GRANAT

Docteur en Sciences Odontologiques, Membre associé de l'Académie Nationale Chirurgie Dentaire,

Chercheur associé au Muséum national

d'histoire naturelle,

UMR 5145; 5198.

Histoire naturelle de l'Homme préhistorique

Musée de l'Homme,

CNRS,

Paris.

\section{Roland BENOÎT}

Docteur d'État en Odontologie,

Orthodontiste (Paris),

Membre titulaire de I'Académie Nationale

chirurgie dentaire,

commission de la Recherche.

Professeur à l'Université Descartes (Paris V)

et Claude Bernard (Lyon).

\section{Évelyne PEYRE}

Chargée de Recherche au CNRS,

Muséum national d'histoire naturelle, UMR 5145

Éco-anthropologie et ethnobiologie,

Muséum National d'histoire Naturelle,

Musée de l'Homme,

CNRS,

Paris.

La canine est une dent différente des autres dents depuis le début de la lignée mammalienne.

L'étude de son évolution est riche d'enseignement pour la distinction des espèces. Très développée dans certaines familles animales actuelles elle était de faibles dimensions chez les premiers mammifères. Chez les Primates, de faible hauteur à l'origine, la canine s'est spécialisée en croc chez les singes actuels. En revanche, chez les Hominidés la canine ne dépasse pas le niveau des dents voisines et est en contact avec elles. II n'y a pas de diastèmes. La génétique du développement permet de suivre l'évolution morphologique et le nombre des dents dans chaque lignée. Elle remonte jusqu'aux reptiles mammaliens, premiers promammifères chez lesquelles la canine a déjà sa morphologie actuelle et est unique par hémi-mâchoire.

- canine

- évolution animale

- reptiles

- mammifères placentaires
AOS 2008:244:309-316

DOI:10.1051/aos:2008042

(C) AEOS / EDP Sciences 


\section{Introduction}

$>$ Aujourd'hui, en odontostomatologie, la canine est considérée comme une dent très importante et digne d'un grand intérêt.

Elle est considérée comme "une dent à part» ou «dent fascinante, pas comme les autres, singulière» et l'implantologie s'intéresse à son remplacement.

Pour bien connaître cette dent, il faut plonger très loin dans le temps à la recherche de ses origines et loin dans l'Histoire des Sciences pour savoir depuis quand et pourquoi cette dent a été nommée «canine».

"Canine», en tant que substantif, désigne une dent. Les dents sont généralement classées en incisives, prémolaires, molaires et une canine par hémi-maxillaire. En effet, tous les autres groupes de dents sont généralement composés de plusieurs éléments. Au cours de l'évolution des mammifères, les groupes incisif, prémolaire et molaire, en se spécialisant se sont réduits en

\section{Historique}

De nombreux termes d'anatomie dentaire, en français, datent du $x \mathrm{VI}^{\mathrm{e}}$ siècle avec le renouveau de l'anatomie et des dissections. "Dent canine» daterait de 1541, signifie «dent du chien» et a été utilisé pour désigner la dent «pointue» de l'Homme et des animaux. Le terme "Canine», employé seul pour la dent humaine, se retrouve en 1546, dans "Fuvres médicales» de Charles Estienne, livre daté de 1545-1561. C'est cette définition que nous retrouvons aujourd'hui dans les dictionnaires et les encyclopédies.

Jusqu'alors, l'enseignement médical se faisait en latin ou en grec. Tous les ouvrages étaient des nombre, pour parfois ne comporter aucun élément. Leur morphologie s'est modifiée selon le mode de vie de l'animal, son environnement, de même que le nombre de leurs tubercules. La canine est restée unique ou a disparu mais sa forme conique, au tubercule principal développé et pointu s'est perpétuée au cours des centaines de millions d'années de son évolution. La canine évoque le mystère, la peur, depuis les représentations des vampires, ces chauve-souris carnivores aux canines acérées et qui sucent le sang humain et, depuis celles de Dracula avec des canines démesurément longues et pointues. La canine serait-elle une dent différente des autres dents ou une dent normale?

Nous allons essayer de suivre l'évolution de la canine depuis sa première apparition au sein du monde animal, il y a fort longtemps, et de savoir d'où vient ce nom «canine» dans la langue française depuis des temps relativement très récents.

manuscrits. L'imprimerie, découverte au $x v^{e}$ siècle, va contribuer à augmenter le nombre des ouvrages qui seront publiés dans leur langue d'origine. L'idée de les traduire ou d'en écrire d'autres en français, pour élargir l'enseignement de la médecine, se développe au $x v{ }^{\mathrm{e}}$ siècle avec Ambroise Paré qui ne connaissait pas le latin. Les noms latins ou grecs vont ainsi être transposés en français ou s'il n'y en avait pas, vont être créés. En dehors de la morphologie coronaire observable, les dents sont peu ou très mal connues. Deux anatomistes vont avancer des idées révolutionnaires (Granat, Peyre 2004). 
Gabriel Fallopio (1523-1562), contrairement aux autres idées émises en ce temps-là, affirme que : «Les dents permanentes et lactéales ont chacune leur alvéole qui s'édifie en même temps que la dent et que les dents permanentes antérieures remplacent les dents de lait.» II est le premier à soutenir que la calcification des dents commence in utero. On en doutera jusqu'au xVIII siècle et on dira qu'il y a indépendance des deux dentures. II réfute la théorie appendiculaire. Douze dents sont édifiées in utero dans chaque mâchoire. Mais, il reste convaincu que les dents sont des os.

Bartolomeo Eustachio (v. 1510-1574) a écrit le premier livre d'anatomie dentaire, en 1564, et démontré que les dents ne sont pas de l'os mais composées d'émail et d'ivoire. II décrit aussi les racines des dents de lait, mais ne parle pas de leur résorption. Les dents sont insérées dans les gencives comme les ongles dans la peau. La minéralisation se fait de la couronne vers la racine.

II faudra attendre le XVIII ${ }^{e}$ siècle pour une meilleure connaissance des dents, mais il restera acquis que les canines sont des dents pointues évoquant celles du chien.

\section{Quelques définitions}

Nous avons retenu celle de l'encyclopédie en ligne, Wikipédia qui est très complète.

«Les canines sont des dents pointues, parfois très saillantes chez certains animaux, situées entre les incisives et les molaires. Elles servent à arracher la nourriture chez les carnivores (par exemple les chats). On les appelle parfois les crocs. Les canines tirent leur nom du mot latin canus, le chien. Chez les humains, les canines sont au nombre de quatre: deux supérieures, deux inférieures. Elles sont absentes chez les espèces herbivores. Les canines ont un rôle très important. Ce sont des dents très solides, avec une racine très longue (jusqu'à 3 centimètres). Leur rôle est primordial dans l'occlusion, lors des mouvements de mastication. Ce sont elles qui prennent en charge les forces dans les mouvements de latéralité; elles doivent entraîner la désocclusion des autres dents. La tradition fantastique attribue des dents pointues aux vampires pour sucer le sang».

Dans le dictionnaire de la langue française d'Émile Littré, publié à partir de 1863 nous trouvons :

«Canin, ine, adjectif pouvant être utilisé comme substantif. Dents canines (angulaires, conoïdes, ou cillères) celles qui sont placées entre les molaires et les incisives. Les canines servent à rompre et à briser les corps durs.»

Nous pouvons résumer les nombreuses définitions ainsi :

Canine, dent pointue placée entre les molaires et les incisives, aux coins de la bouche. Les canines, doivent leur nom à leur ressemblance avec les crocs des chiens, dents très pointues. Elles sont coniques, et la pointe de leur couronne s'élève parfois au-dessus du niveau des autres dents. Les canines permettent de déchirer la nourriture qui est ensuite mastiquée par les autres dents.

Rappelons aussi que la canine maxillaire a été appelée «dent de l'œil» car, avant les thérapies modernes la cellulite secondaire à une infection de la canine pouvait induire une thrombophlébite faciale voire craniofaciale. Un syndrome septicémique sévère avec un œdème important gagne l'angle interne de l'œil et la paupière supérieure. La veine faciale est thrombosée entrainant une atteinte oculaire et surtout une atteinte méningée par propagation de l'infection le long de la veine angulaire de l'œil. 
C'est donc avec cette notion de la canine que les chercheurs en zoologie, en biologie, en anatomie comparée, en sciences naturelles, ont entrepris leurs recherches depuis le XvII siècle.

Nous savons aujourd'hui que la diversité animale date du début de l'Ère primaire (540 millions d'années) au cours de laquelle différents invertébrés et vertébrés occuperont les mers. Les premiers vertébrés, des poissons, sont nos plus lointains ancêtres. La sortie de l'eau par les amphibiens et la conquête de la terre ferme apparaissent à la fin de l'Ère primaire vers 320 millions d'années. Ils donneront naissance aux reptiles primitifs.

\section{Évolution de la canine}

L'Homme moderne possède 32 dents dont 16 réparties régulièrement sur les maxillaires et 16 sur la mandibule. Par hémi-arcade, on compte donc 8 dents dont une canine. Celle-ci effectivement est la dent un peu conoïde, située entre les 2 incisives antérieures et les 2 prémolaires et 3 molaires distales. Chez l'Homme, ces dents sont agencées en série continue, sans diastèmes. Les 2 arcades alvéolo-dentaires sont de forme elliptique.

Dans le règne animal, le nombre des dents varie d'un genre, d'une famille, voire d'une espèce à l'autre, mais il y a toujours le même nombre de dents, du côté gauche et du côté droit. En revanche, il n'en est pas toujours de même entre maxillaire et mandibule (Granat et al, 1998).

Depuis quand I'Homme moderne a-t-il 32 dents et depuis quand les dents ont-elles leur morphologie actuelle ? Pour répondre à cette question il nous faut placer l'Homme au sein du Monde animal.

\section{Évolution de l'Homme}

L'Homme est un Primate. Tous les Hommes actuels appartiennent à la même et unique espèce, Homo sapiens. Toutes les autres espèces d'Hominidés se sont éteintes au cours du temps. Les Primates sont des mammifères placentaires.
L'histoire de nos dents va donc nous faire remonter très loin en arrière jusqu'aux premiers mammifères.

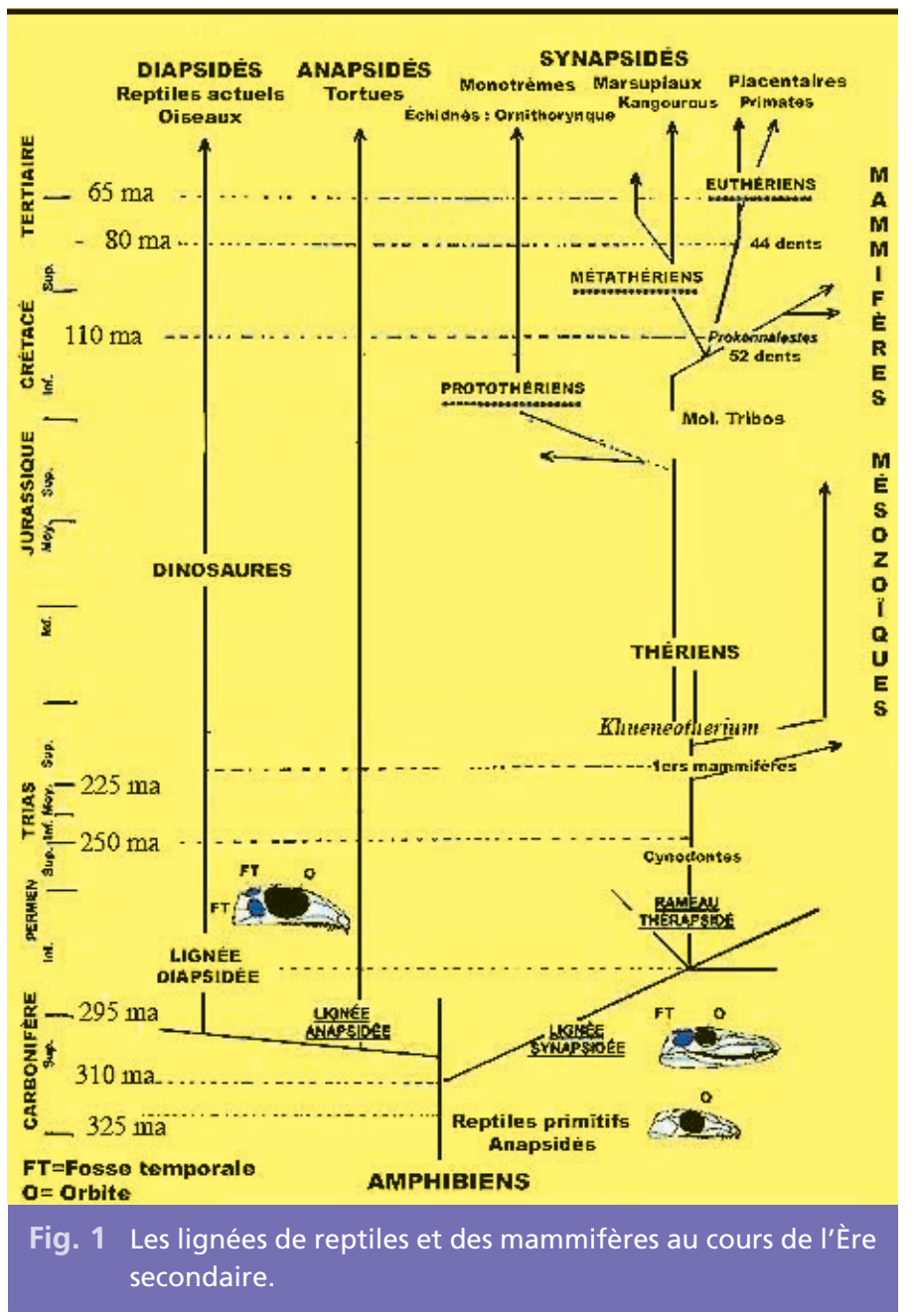


Les premiers reptiles, très primitifs, apparaissent donc vers 320 millions d'années, avant aujourd'hui (fig. 1). Ils ne possèdent pas de fosse temporale. On les dit "Anapsidés». Vers 310 millions d'années, de ce tronc reptilien, va se détacher une première grande branche celle des "Synapsidés » avec une seule fosse temporale. Nous avons aussi une seule fosse temporale par hémi-côté. Ils sont donc nos très lointains ancêtres. 10 millions d'années plus tard, vers 300 millions d'années, une seconde branche se détache. Elle donne rapidement naissance au rameau des tortues qui restent Anapsidés, puis mène à tous les autres reptiles qui possèdent deux fosses temporales, superposées, ils sont «Diapsidés». Ce sont les grands reptiles Dinosauriens de l'Ère Secondaire et les survivants de la grande extinction de la fin du Secondaire, les reptiles actuels et les oiseaux. Aucun reptile diapsidé n'est notre ancêtre (Sigogneau - Russel 1991, 1994).

Chez les Reptiles diapsidés, les dents sont en nombre élevé (polyodontie), toutes à peu près semblables (isodontie), de forme conique (haplodontie), à rôle essentiel préhensile (Anthony 1973). Elles sont remplacées de façon illimitée (polyphyodontie) et sont fixées à leur support osseux par soudure à leur base (acrodontie) ou par ankylose sur leur face vestibulaire (pleurodontie).

Les dents des reptiles actuels sont de même (fig. 2). Seuls les oiseaux n'ont pas de dents. Notre rameau est séparé de ce rameau reptilien depuis 300 millions d'années. Revenons à la première branche. Vers 280 millions d'années, les Synapsidés donnent naissance au rameau des Thérapsidés, appelés aussi Reptiles mammaliens. Leur fosse temporale unique est maintenant bordée inférieurement par l'arcade zygomatique. Disposition qui se perpétuera jusqu'à nos jours chez les mammifères et

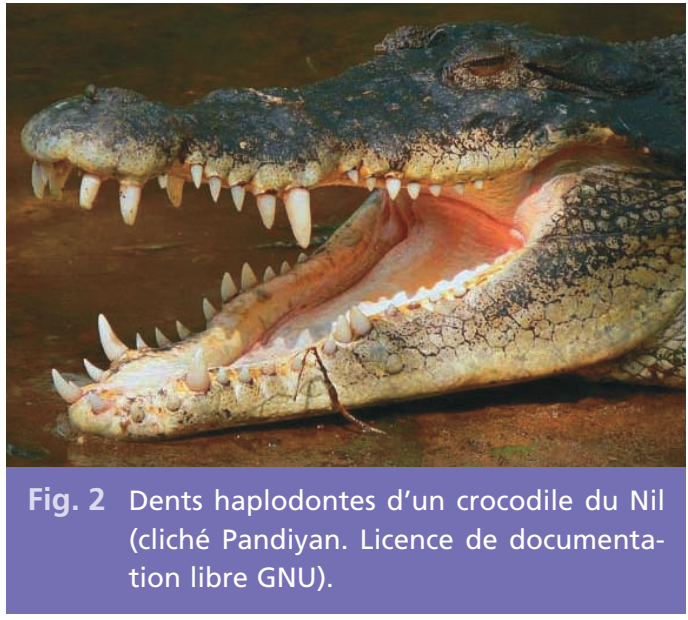

l'Homme. Les dents simples, coniques, sont déjà de véritables organes dentaires, constitués de tissus durs (émail, dentine), de tissus mous (pulpe coronaire et radiculaire), composés d'une couronne visible et d'une racine enchâssée dans l'os maxillaire: c'est la Thécodontie.

Les plus évolués de ces Thérapsidés, les Cynodontes se reconnaissent vers 250 m.a, à l'aube de I'Ère secondaire. Ils présentent de nombreux caractères évolués dont un palais dur qui va séparer la cavité nasale de la cavité buccale. Ils sont de plus en plus considérés comme les ancêtres directs des mammifères qui eux allaitent leurs petits et apparaissent vers 210 million d'années. Le nombre de dentitions de remplacement se réduit. Lorsqu'il n'en reste que deux, Diphyodontie, la lactéale et la permanente, le stade évolutif est semblable à celui des Mammifères. On observe que la surface occlusale des post-canines augmente et se complique, par adjonction de tubercules nouveaux (plexodontie). La dent porte alors trois tubercules disposés sur une même ligne mésio-distale, le central étant le plus élevé, c'est le type triconodonte (Granat et al, 1992). 


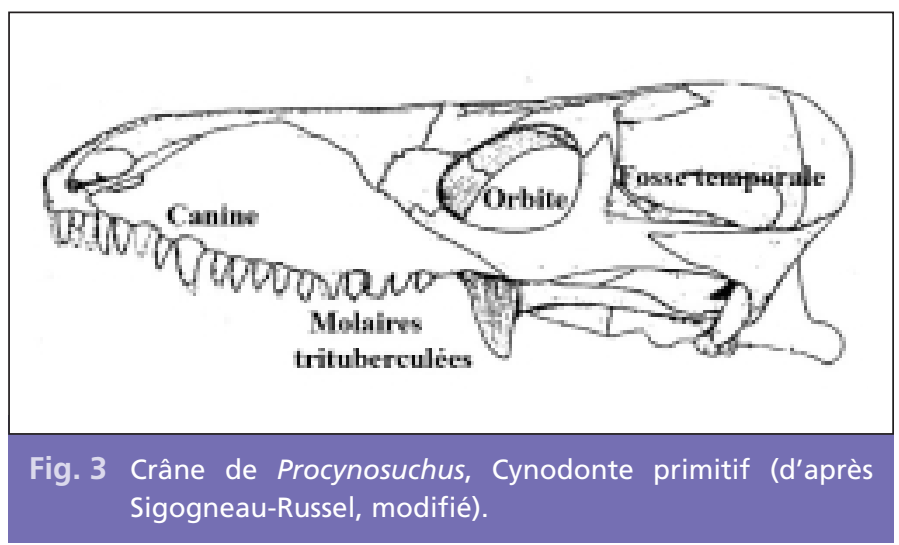

Chez les Cynodontes (fig. 3), la canine est la première dent à se différencier. Chez certains elle devient forte, conique et pointue. La canine supérieure se définit comme la première dent portée par le maxillaire. La canine inférieure est la dent qui, en occlusion, se place juste en avant de la supérieure. L'Homme moderne a conservé cette disposition primitive. D'où l'origine du nom «cynodonte», reptile qui a des dents de chien (les canines). Nous voici au début de l'Histoire des dents humaines. Les éléments dentaires antécanines sont implantés dans les prémaxillaires. Ils seront les incisives, très vite au nombre de quatre. Le nombre des post-canines (prémolaires et molaires) va diminuer à 9. Les cynodontes ont formé de très nombreux groupes. II est possible que les ancêtres réels des mammifères de petites formes soient des cynodontes primitifs qui sont restés de petite taille, alors que la plupart des Cynodontes se sont spécialisés en différents genres, en augmentant de taille et avec des canines en crocs, puis se sont éteints.

Des 3 grands rameaux de mammifères de l'Ère secondaire (mésozoïque), celui des Thériens, mammifères vrais, donnera naissance aux mammifères actuels.
Chez les mammifères Thériens, depuis 200 millions d'années avec Kuehneotherium, les trois tubercules principaux des molaires sont disposés en triangle (trigonodonte). Disposition que nous avons conservée (Kielan-Jaworowska et al, 1980, 1989).

À chaque niveau évolutif, les espèces sont nombreuses et se spécialisent différemment, d'où la grande variabilité morphologique des dents dès cette époque. Sur le rameau Thérien vers 110 m.a. apparaît la branche des Euthériens ou Placentaires, la nôtre. Parturition et allaitement sont acquis. Chez eux, les canines sont de petite taille et ne dépassent guère le niveau des autres dents. L'un des plus anciens Placentaires connu à ce jour, notre ancêtre, nommé Prokennalestes, du désert de Gobi, en Mongolie, est daté de 110 millions d'années environ (fig. 4). Il est de très petite taille. Sa formule dentaire est à 52 dents (20 de plus que la nôtre), avec par hémi-mâchoire, 4 incisives spatulées, 1 seule canine de petite taille d'après l'alvéole, 5 prémolaires portant un ou plusieurs petits tubercules supplémentaires et seulement 3 molaires, caractère des Placentaires. Voilà encore une formule ancestrale avec toujours une seule canine conique, pointue. Vers 80 millions d'années se reconnaissent 

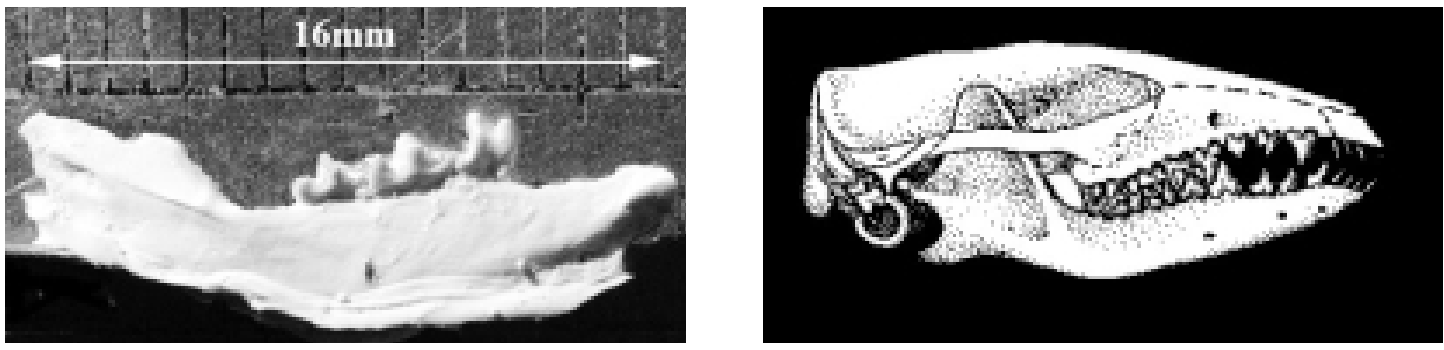

Fig. 4 À gauche, mandibule de Prokennalestes. Seules 3 molaires sont présentes (cliché J. Granat). À droite, crâne d'Asiorictes Mammifère placentaire de la fin de l'Ère secondaire. Sa canine est de même taille que ses 3 es prémolaires (d'après Sigogneau-Russel, modifié).

les ancêtres directs de tous les placentaires qui vont apparaître ensuite.

Ce sont des mammifères de petite taille, celle d'une souris. Leur formule dentaire était à 44 dents, avec toujours, par hémi-mâchoire, une seule canine, mais 3 incisives, 4 prémolaires et 3 molaires. Les incisives sont aplaties vestibulolingualement, la canine est relativement de petite taille, conique et pointue et son sommet ne dépasse pas exagérément celui des dents voisines, tel Asiorictes du désert de Gobi (fig. 4). A l'origine, elle n'a donc rien d'un croc. Les deux prémolaires antérieures sont simples et les 2 postérieures à plusieurs tubercules plus ou moins développés. Les 3 molaires à 6 tubercules sont en série décroissante, la première molaire est la plus volumineuse. C'est la disposition primitive que l'on retrouve chez I'Homme moderne. Ces ancêtres étaient omnivores voire insectivores. Pendant 20 millions d'années la canine est restée à sa place, conique et pointue mais de petite taille chez les insectivores, développée en croc chez les carnivores.

À la fin de l'Ère secondaire, au Crétacé supérieur, une grande extinction touche le monde animal et pas seulement les Dinosaures. De nombreuses niches écologiques étant inhabi-

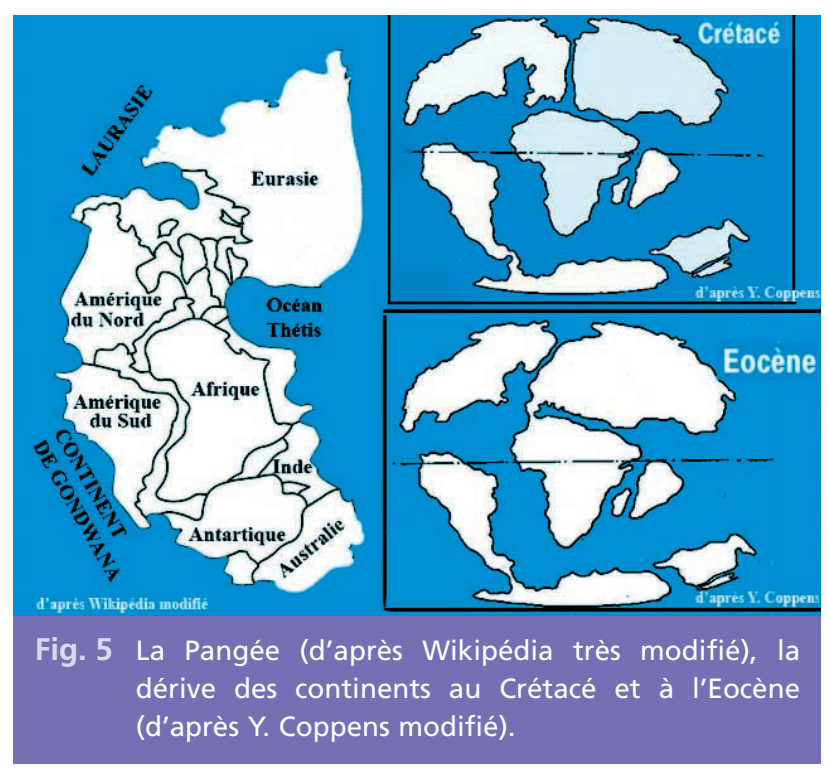


tées, les mammifères survivants, de petite taille, insectivores vont connaître une expansion considérable et vont occuper tout l'espace disponible. Ils le partageront avec certains reptiles de petite taille, des oiseaux volants et des formes coureuses (Autruches). Rappelons aussi que les continents n'avaient pas l'aspect que nous leur connaissons aujourd'hui. Jusque là, la Pangée regroupait 2 grands continents, le Gondwana et la Laurasie séparés par une mer, I'océan Thétis (fig. 5). À la fin du Secondaire, la dérive des continents se met en route et tous les continents vont bouger dès le début du Tertiaire, à l'Eocène, se rapprocher ou s'éloigner comme I'Amérique, emportant les animaux qui y vivaient (Coppens 1983).

\section{Ouvrages à consulter}

Anthony J.

Cours d'anatomie dentaire

comparée.

Prélat. Paris 1973;126 p.

Coppens $Y$.

Le Singe, I'Afrique et l'Homme.

Fayard, éd., Paris 1983.

Granat J., Genet-Varcin E.,

Heim J. L.

Évolution de la denture permanente des hominidés.

Encycl. Méd. Chir., Stomatologie

et Odontologie 1992;22003,

S10:11 p.

Granat J., Heim J. L.

Histoire naturelle de la formule dentaire humaine.

In: Biom. Hum. et Anthropol. 16,

1-2. C.N.R.S. Paris 1998;1-12.

Granat J., Peyre E.

L'Homme, ses incisives,

son évolution

et l'anatomie cranio-faciale

au $x v I^{e}$ siècle.

Biom. Hum. et Anthropol

2004;21, n 3-4, 135-143.

Kielan-Jaworowska Z.

Les premiers mammifères.

In : La Recherche $\mathrm{N}^{\circ} 108$,

1980;11:146-155.

Kielan-Jaworowska Z.,

Dashzeveg D.
Eutherian mammals from

the Early Cretaceous

of Mongolia.

In : Zoologica Scripta 1989;

Vol.18 No2, pp. 347-355.

Sigogneau-Russel D.

Les Mammifères au temps des dinosaures.

Masson. éd., Paris 1991;199 p.

Sigogneau-Russel D.

Les Mammifères dans la nuit

des temps mésozoïques.

La Vie des Sciences,

C.R.Acad.Sci., série générale

1994;11(4):243- 270.

\section{SUMMARY}

\section{The canine.}

\section{First part: Definition and evolution during the Secondary Era}

Jean Granat

Roland Benoît

Évelyne Peyre

$$
\begin{aligned}
& \text { Keywords } \\
& \text { - canine } \\
& \text { - animal evolution } \\
& \text { - reptiles } \\
& \text { - mammals placental }
\end{aligned}
$$

The tooth canine is different from other teeth since the beginning of the mammalian lineage.

The study of evolution is instructive to distinguish species. Highly developed in some present animal families, it was small in early mammals. Among Primates, low height at the origin, today, the canine is specialized in a fang in monkeys families. In return, the Hominids canine does not exceed the level of adjacent teeth and is in contact with the neighboring teeth. There is no diastema. The genetics of development can follow the morphological evolution and the number of teeth in each line. It goes back to mammalian reptiles, first promammals (Cynodonte) in which the canine present a modern morphology and is single in half-jaw. 